\title{
DESIGN OF POWER-SPLIT HYBRID VEHICLES WITH A SINGLE PLANETARY GEAR
}

\author{
Chiao-Ting Li \\ Department of Mechanical \\ Engineering \\ University of Michigan \\ Ann Arbor, MI, 48109 \\ ctli@umich.edu
}

\author{
Xiaowu Zhang \\ Department of Mechanical \\ Engineering \\ University of Michigan \\ Ann Arbor, MI, 48109 \\ xiaowuz@umich.edu
}

\author{
Huei Peng \\ Department of Mechanical \\ Engineering \\ University of Michigan \\ Ann Arbor, MI, 48109 \\ hpeng@umich.edu
}

\begin{abstract}
This paper presents a systematic design methodology for split hybrid vehicles using a single planetary gearset (PG) as the transmission. The design methodology consists of four steps: 1) analyze clutch locations on the $P G$ and operation modes, 2) generate dynamic models, 3) evaluate drivability (acceleration performance) via forward simulations, and 4) optimize the fuel economy using the dynamic programming technique. The 1-PG split hybrid transmission can have 12 configurations, and each configuration can have four operation modes when three clutches are added. This methodology systematically evaluates all configuration candidates and identifies the optimal design, and we demonstrate how it helps to identify a simplified design based in the output-split configuration used by the Chevy Volt. The simplified design, named the Volt, has only two of the four operation modes of the original Volt. The Volt achieves the same fuel economy as the original Volt in the FUDS cycle, and has only slightly reduced drivability and fuel economy in the HWFET cycle. In addition, an improved design based on the input-split configuration used by the Toyota Prius is also identified, named the Prius ${ }^{+}$, which has one additional mode than the original Prius. The Prius ${ }^{+}$outperforms the Prius in both drivability and fuel economy.
\end{abstract}

\section{NOMENCLATURE}

$\omega$ Rotational speed of powertrain component

$\dot{\omega}$ Rotational acceleration of powertrain component

$T$ Torque of powertrain component

$I \quad$ Inertia of powertrain component

$F \quad$ Internal force between gears on the planetary gearset
$K \quad$ Final drive gear ratio on output shaft

$R \quad$ Gear radius of the ring gear

$S$ Gear radius of the sun gear

$e$ (subscript) Engine

$M G$ (subscript) Electric machine

out (subscript) Output shaft

\section{INTRODUCTION}

The power-split configurations have dominated the hybrid electric vehicles (HEVs) markets in the US and in the worldmore than $90 \%$ of the hybrid vehicles sold in 2010 are powersplit type [1,2]. Currently, most of the production models use a single planetary gearset $(\mathrm{PG})$ as the transmission, including the Toyota Prius and the Chevy Volt. However, these two vehicles are very different; the Prius is an input-split HEV (an electric machine is connected to the output shaft) with only one operation mode and no clutch is used. The Volt, on the other hand, is an output-split HEV (an electric machine is connected to the engine) with three clutches to allow four operation modes. These differences indicate that there are many design decisions to be made even when only one PG is used. Earlier studies have shown that when two PGs are used, there are 1,152 possible configurations [3], and when one PG is used, there are 12 possible configurations [4]. Adding clutches on the PG allows the same configuration to have multiple operation modes; it has found that a 1-PG configuration can have as many as four useful modes by adding three clutches [4]. When the basic configurations are determined, vehicle performance, such as the speed/torque characteristics of the powetrain components, vehicle weight, driving patterns, vehicle acceleration performance and fuel economy, must be 
considered in the design process. For example, the optimal 2PG split configurations for a combat vehicle with demanding launching requirement [3] and the optimal configuration for a delivery truck designated to city driving [5] are found to be different, through a design process which includes searches for configuration, powertrain component sizing and optimal controls for fuel economy. The candidate pool of the split configuration is large and there are many factors affecting the vehicle performance; thus, identifying the optimal design cannot be done merely based on engineering intuitions or heuristic judgments. The main contribution of this paper is the development of a systematic design methodology to thoroughly explore and screen through the candidate pool, and the various performance related factors will be taken into account comprehensively to identify the optimal design. The focus will be on the 1-PG design case as most of the power-split hybrid vehicles currently on the market use a single planetary gear because of the simple design and lower packaging requirement.

Generic models that are capable of representing dynamics of the power-split powertrain and can be reconfigured to represent all the configurations in the design pool are needed. Dynamics of the Prius configuration have been well studied [6, 7] but they only describe that particular configuration. Kinematics of PGs have been reported in the literature [8], and the lever diagram providing a graphical representation has been widely used [9]. Parametric studies using the lever diagram for dynamics of the 2-PG system has also been reported [10, 11], which is one way to generalize the dynamic models of powersplit configurations. Applying permutations on the lever diagram is another way to generate generic representations for all split configuration candidates and has been used in several studies [3-5]. Furthermore, screening rules to reduce the population in the design candidate pool have been reported in [3] for HEVs and [5] for hydraulic hybrid vehicles; in particular, [5] has included rules to exam the speeds of powertrain components and drivability (i.e. acceleration performance) as additional screening criteria.

Optimal power management strategy for HEV driving is crucial in the design process because the design decision should be made based on the best-execution performance of each design candidate. Often, fuel consumption is taken as the objective function; however, other performance attributes can be included, such as battery state of charge (SOC) fluctuation or emissions. Rule-based controls [6, 12, 13], the Equivalent Consumption Minimization Strategy (ECMS) [14-16], the Pontryagin's minimum principle [17-19], and the dynamic programming (DP) techniques [20-22] have all been used to design power management algorithms of HEVs. Among them, only the DP technique guarantees global optimality over the problem horizon. Therefore, DP has seen increasing application in recent years. A summary explaining and comparing different HEV power management algorithms can be found in [23].
The design methodology proposed in this paper thoroughly explores the 1-PG slit configuration with multiple clutches and operational modes in four steps: 1) analyze clutch locations on the PG and operation modes, 2) generate dynamic models, 3) evaluate drivability, and 4) optimize the fuel economy. Iterating through these steps is needed to finalize the design - including elimination of unnecessary clutches, selection of design parameters, and optimal control strategy. The rest of this paper is organized as follows: the analysis on a single PG will first be presented; followed by the explanation of the four steps of the design process. The design process will be illustrated on two particular configuration candidates: the output-split configuration used by the Chevy Volt and the input-split configuration used by the Toyota Prius. However it is important to note that the process can be used on all 1-PG configurations. Finally, concluding remarks are provided.

\section{ANALYSIS OF SINGLE PLANETARY GEARSET}

Figure 1 shows the schematic diagram of a PG and its lever diagram analogy. The ring gear, sun gear and carrier gear are shown as nodes on the lever diagram, which conveniently visualizes the speed relationship among the gears. The speed relationship is also shown in Eq. (1).

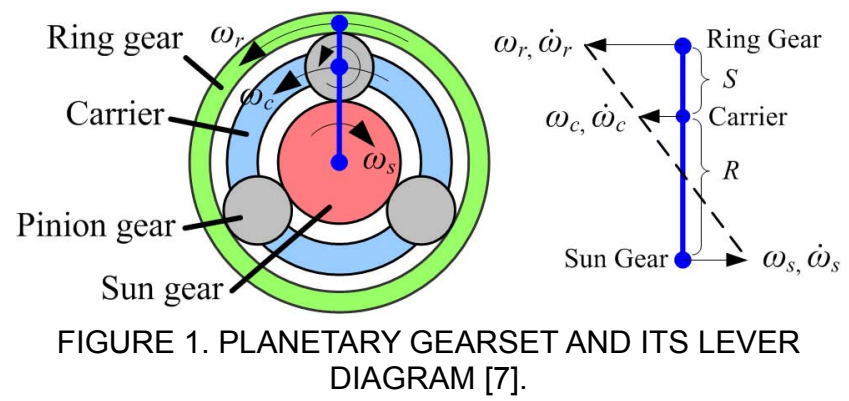

$$
\omega_{s} S+\omega_{r} R=\omega_{c}(R+S)
$$

where the subscript $s$ denotes the sun gear, $r$ denotes the ring gear, and $c$ denotes the carrier, which connects all the pinion gears together. Equation (1) further leads to the rotational acceleration relationship on the PG, as shown in Eq. (2), which is relevant to drivability evaluation in the design methodology.

$$
\begin{aligned}
& \left(\omega_{s}+\dot{\omega}_{s} \Delta t\right) S+\left(\dot{\omega}_{r}+\dot{\omega}_{r} \Delta t\right) R \\
& \quad=\left(\dot{\omega}_{c}+\dot{\omega}_{c} \Delta t\right)(R+S) \\
& \Rightarrow \dot{\omega}_{s} S+\dot{\omega}_{r} R=\dot{\omega}_{c}(R+S)
\end{aligned}
$$

Assuming that the HEV has two electric machines (motor/generator), there will be a total of four powertrain components (the engine, two electric machines, and the output shaft connected to the driven wheels) that need to be assigned to the three nodes on the PG (i.e. connected to the PG). To have a feasible power-split configuration, the degree of freedom (DOF) of the PG must be equal to or less than two, which leads to six input-split configurations and six output-split 
configurations. The permutation starts with assigning the engine, output shaft and one electric machine to the three PG nodes $\left(P_{1}^{3} \times P_{1}^{2} \times P_{1}^{1}=3 ! \times 2 ! \times 1 !=6\right)$. The second electric machine is then assigned to either the output shaft (which produces an input-split configuration) or the engine (which produces an output-split configuration), so the candidate pool size of the 1-PG designs is 12 .

\section{SYSTEMATIC DESIGN PROCESS}

Figure 2 summarizes the four steps of the proposed design process. These four steps are explained in the following subsections. We will use the output-split configuration used by the Chevy Volt as an example to explain the design process. However, the same procedure can be applied to all 12 configuration candidates.

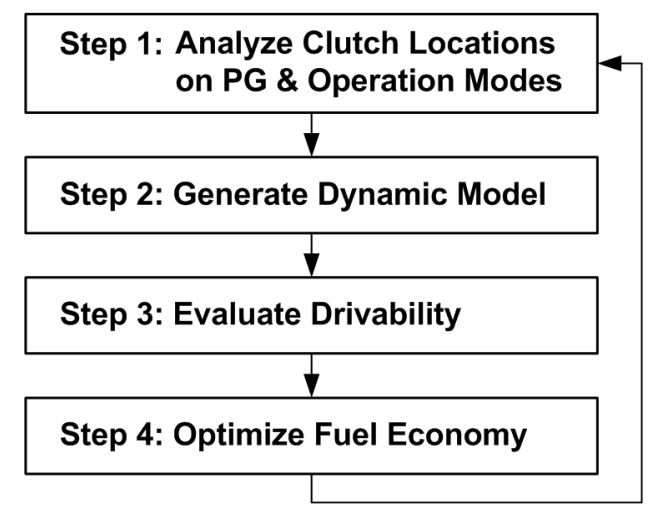

FIGURE 2. FLOWCHART OF THE DESIGN PROCESS.

\section{Step 1: Analyze Clutch Locations on PG and Operation Modes}

Figure 3 shows the output-split configuration used by the Chevy Volt with the seven possible locations for adding clutches, but notice that the Volt actually has only the three clutches shown in the green color.

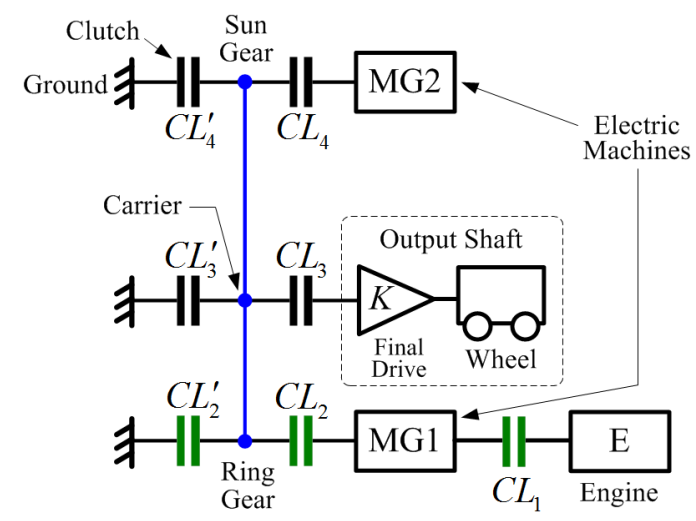

FIGURE 3. THE OUTPUT-SPLIT CONFIGURATION USED BY THE CHEVY VOLT WITH ADDITIONAL CLUTCHES.

The clutch, $C L_{1}$, is used to connect/disconnect the engine to MG1 (the first electric machine). The other clutches are grouped into pairs. For example, $C L_{2}$ and $C L_{2}$ ' is a pair, and their clutch states are complimentary. In other words, when one of them is open, the other must be closed, and vice versa (i.e. they cannot be both open or both closed). This applies to the other two clutch pairs $C L_{3} \& C L_{3}^{\prime}$, and $C L_{4} \& C L_{4}$ '. Furthermore, the clutch pair $C L_{3} \& C L_{3}$ ' is judged to be unnecessary, because we assume that the output shaft is always connected to the PG and the standing-still battery charging never happened. Therefore, in the following, we will focus only on the other five clutches, $C L_{1}, C L_{2} \& C L_{2}{ }^{\prime}$, and $C L_{4} \& C L_{4}$ '.

Table 1 lists the eight possible operation modes that can be generated by the abovementioned five clutches. The analysis in [4] has shown that only the first four modes are useful; more specifically, Mode $1\left(\mathrm{EV}_{1}\right)$ and Mode $2\left(\mathrm{EV}_{2}\right)$ are pure-electric modes; Mode 3 is a series mode; and Mode 4 is a split mode. The other four modes are either infeasible or redundant. The four useful modes, shown in Figure 4, are the four modes the Chevy Volt has, which can be achieved by the three green clutches (the clutch pair $C L_{4} \& C L_{4}{ }^{\prime}$ is unnecessary because MG2 is always connected to the PG).

TABLE 1. CLUTCH STATES \& OPERATION MODES OF THE OUTPUT-SPLIT CONFIGURATION USED BY CHEVY VOLT.

\begin{tabular}{lccc}
\hline \multicolumn{1}{c}{ Mode } & $\boldsymbol{C L}_{\mathbf{1}}$ & $\boldsymbol{C L}_{\mathbf{2}}$ & $\boldsymbol{C L}_{\mathbf{4}}$ \\
\hline $1\left(\mathrm{EV}_{1}\right)$ & 0 & 0 & 1 \\
\hline $2\left(\mathrm{EV}_{2}\right)$ & 0 & 1 & 1 \\
\hline 3 (Series) & 1 & 0 & 1 \\
\hline 4 (Split) & 1 & 1 & 1 \\
\hline 5 (Infeasible) & 0 & 0 & 0 \\
\hline 6 (= EV $\left.{ }_{1}\right)$ & 0 & 1 & 0 \\
\hline 7 (Infeasible) & 1 & 0 & 0 \\
\hline 8 (No EVT) & 1 & 1 & 0
\end{tabular}

Description: "0" means the clutch is open; "1" means the clutch is closed.

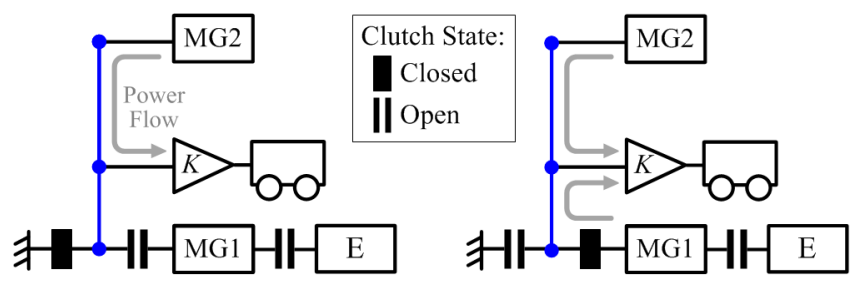

(a) Mode $1\left(\mathrm{EV}_{1}\right)$ (b) Mode $2\left(\mathrm{EV}_{2}\right)$

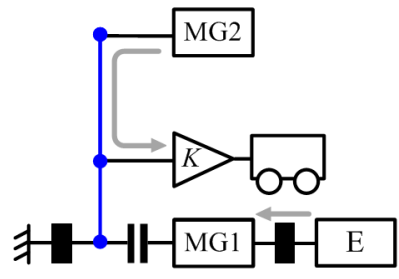

(c) Mode 3 (Series)

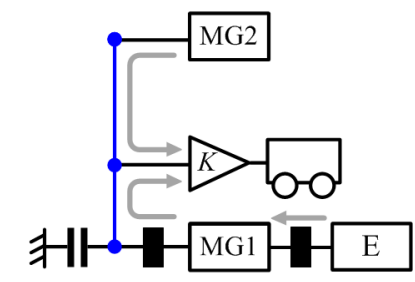

(d) Mode 4 (Split)
FIGURE 4. THE FOUR OPEARTION MODES OF CHEVY VOLT 


\section{Step 2: Dynamic Model Generation}

With the clutch states specified, the dynamic equations can be generated for each of the operation modes. Equations (3)-(6) are the state space representations of the four useful operation modes shown in Figure 4, which consist of rotational dynamics of each powertrain component, and the internal force acting between gears on the PG is also included in the equation. Notice that not all operation modes require four equations to describe the dynamics; equations of disconnected powertrain components are irrelevant and can be eliminated.

Mode 1:

$$
\left[\begin{array}{ccc}
\frac{m r^{2}}{K^{2}} & 0 & R+S \\
0 & I_{M G 2} & -S \\
R+S & -S & 0
\end{array}\right]\left[\begin{array}{c}
\dot{\omega}_{\text {out }} \\
\dot{\omega}_{M G 2} \\
F
\end{array}\right]=\left[\begin{array}{c}
-T_{\text {load }} \\
T_{M G 2} \\
0
\end{array}\right]
$$

Mode 2:

$$
\left[\begin{array}{cccc}
I_{M G 1} & 0 & 0 & -R \\
0 & \frac{m r^{2}}{K^{2}} & 0 & R+S \\
0 & 0 & I_{M G 2} & -S \\
-R & R+S & -S & 0
\end{array}\right]\left[\begin{array}{c}
\dot{\omega}_{M G 1} \\
\dot{\omega}_{\text {out }} \\
\dot{\omega}_{M G 2} \\
F
\end{array}\right]=\left[\begin{array}{c}
T_{M G 1} \\
-T_{\text {load }} \\
T_{M G 2} \\
0
\end{array}\right]
$$

Mode 3:

$$
\left\{\begin{array}{l}
\left(I_{e}+I_{M G 1}\right) \dot{\omega}_{M G 1}=T_{M G 1}+T_{e} \\
{\left[\begin{array}{ccc}
\frac{m r^{2}}{K^{2}} & 0 & R+S \\
0 & I_{M G 2} & -S \\
R+S & -S & 0
\end{array}\right]\left[\begin{array}{c}
\dot{\omega}_{\text {out }} \\
\dot{\omega}_{M G 2} \\
F
\end{array}\right]=\left[\begin{array}{c}
-T_{\text {load }} \\
T_{M G 2} \\
0
\end{array}\right]}
\end{array}\right.
$$

Mode 4:

$$
\left[\begin{array}{cccc}
I_{M G 1}+I_{e} & 0 & 0 & -R \\
0 & \frac{m r^{2}}{K^{2}} & 0 & R+S \\
0 & 0 & I_{M G 2} & -S \\
-R & R+S & -S & 0
\end{array}\right]\left[\begin{array}{c}
\dot{\omega}_{M G 1} \\
\dot{\omega}_{\text {out }} \\
\dot{\omega}_{M G 2} \\
F
\end{array}\right]=\left[\begin{array}{c}
T_{M G 1}+T_{e} \\
-T_{\text {load }} \\
T_{M G 2} \\
0
\end{array}\right]
$$

where $m$ is the vehicle mass, $r$ is the wheel radius, $T_{\text {load }}$ is the load imposed by the rolling resistance and aerodynamic drag during driving and defined at the output shaft.

In addition to modeling the mechanical part of the powertrain dynamics, the battery state of charge (SOC) also need to be modeled. In this paper, the battery is modeled as a voltage source with an internal resistance, and the SOC dynamics are represented by Eq. (7).

$$
S \dot{O} C=-\frac{V_{o c}-\sqrt{V_{o c}^{2}-4 P_{\text {batt }} R_{\text {batt }}}}{2 R_{\text {batt }} Q}
$$

where $V_{o c}$ is the open circuit voltage, $R_{b a t t}$ is the internal resistance of the battery, $Q$ is the battery capacity, and $P_{\text {batt }}$ is the net power drawn out from the battery, which is described by Eq. (8).

$$
P_{\text {batt }}=T_{M G 1} \omega_{M G 1}\left(\eta_{M G 1}\right)^{k}+T_{M G 2} \omega_{M G 2}\left(\eta_{M G 2}\right)^{k}
$$

where $\eta_{M G 1}$ and $\eta_{M G 2}$ are efficiencies of the two electric machines, and $k$ equals to 1 when generating and equals to -1 when motoring.

\section{Step 3: Drivability Evaluation}

Drivability refers to the acceleration performance of a vehicle. For a conventional ICE vehicle, the maximum acceleration can be found by commanding the engine to operate at the maximum torque level. This analogy can be applied to pure-electric and series mode of an HEV because these modes have only one DOF. However, finding the maximum acceleration is more complicated when the HEV is in the split mode because there are multiple powertrain components driving the vehicle. Due to the coupled speed relationship (Eq. (1)), unfortunately, the maximum torques of the powertrain components do not occur simultaneously. Nevertheless, some insight can be extracted from observing the rotational acceleration relationship (Eq. (2)) on the lever diagram.

Figure 5 shows three different ways to produce the same acceleration at the output shaft in Mode 4 (the split mode), and notice that the angular accelerations of all notes on the lever forms the straight dash line, indicating Eq. (2) is satisfied. In Figure 5-(a), the rotational speed of the sun gear will increase quickly due to the large acceleration, and so will the rotational speed of the ring gear in Figure 5-(c). Both cases may lead to limited acceleration performance because constraints on speeds and torques are more likely to become active to limit the torque outputs. In Figure 5-(b), on the other hand, speeds of all powertrain components ramp up moderately, which is more likely to have good acceleration performance without violating the speed/power constraints of any powertrain components.

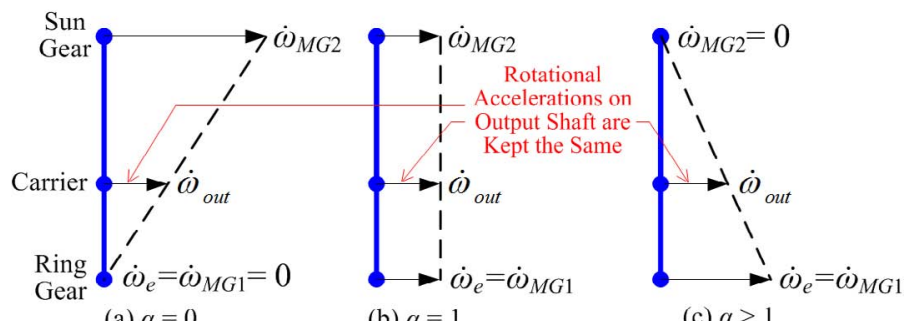

(a) $\alpha=0$

(b) $\alpha=1$

(c) $\alpha>1$

FIGURE 5. DIFFERENT WAYS TO PRODUCE THE SAME VEHICLE ACCELERATION IN THE SPLIT MODE.

The phenomena in Figure 5 can be represented mathematically by the parameterizing relationship shown in Eq. (9), where $\alpha$ is the parameter that relates $\dot{\omega}_{M G 1}$ to $\dot{\omega}_{\text {out }}$; then Figure 5-(a) has $\alpha=0$, Figure 5-(b) has $\alpha=1$, and Figure 5-(c) has $\alpha>1$.

$$
\dot{\omega}_{M G 1}=\alpha \dot{\omega}_{\text {out }}
$$

Figure 6 shows the maximum acceleration with different values of $\alpha$. In addition, we also mark the corresponding active 
constraints when significant drops in acceleration happen. Cases with $\alpha>1$ have the constraint on $T_{M G 1}$ active when the vehicle speed is low, which is consistent with the analysis in Figure 5-(c), in that the ring gear speed will increase quickly and violates the constraint on $T_{M G 1}$. However, the case with $\alpha=$ 0 does not have the constraint on $T_{M G 2}$ (located at the sun gear) becoming active until the vehicle speed is quite high, which is attributed to the wide rotational speed range in MG2. In fact, the case with $\alpha=0$ achieves higher acceleration than the case with $\alpha=1$ when the vehicle speed is in $40-55 \mathrm{mph}$.

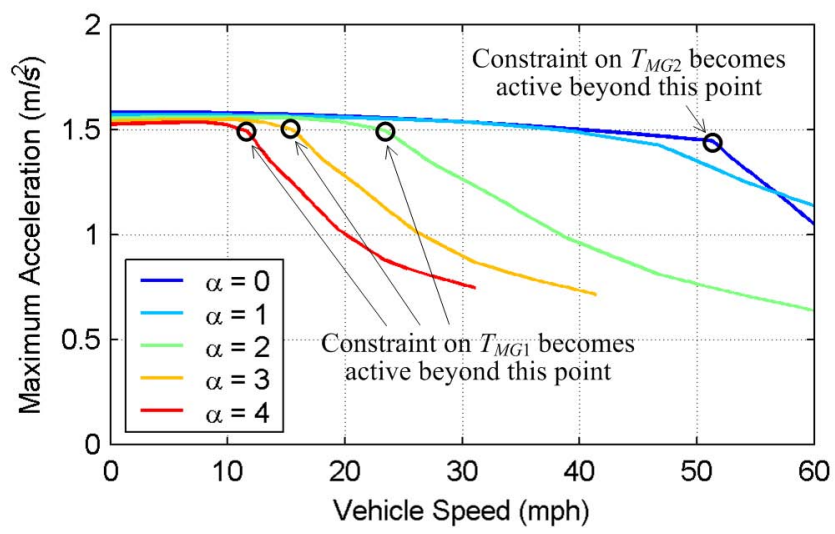

FIGURE 6. MAXIMUM VEHICLE ACCENERATION IN THE SPLIT MODE WITH VARIOUS STRATEGIES

The parametric analysis on the split mode shown above concludes that, to launch the vehicle in this mode, the value of $\alpha$ should be between 0 and 1 . A strategy with $\alpha=0.5$ is applied in a forward simulation to find the shortest time to accelerate the vehicle from 0 to $60 \mathrm{mph}$, and the result is close to the optimal solution obtained by DP, shown in Figure 7. Both solutions indicate that Mode 4 needs about 18 seconds to accelerate from 0 to $60 \mathrm{mph}$. The concept of this parametric analysis can be applied to the split mode operation in all 12 configurations to find strategies (i.e. the parameter $\alpha$ that produces the maximum acceleration) for drivability evaluation.

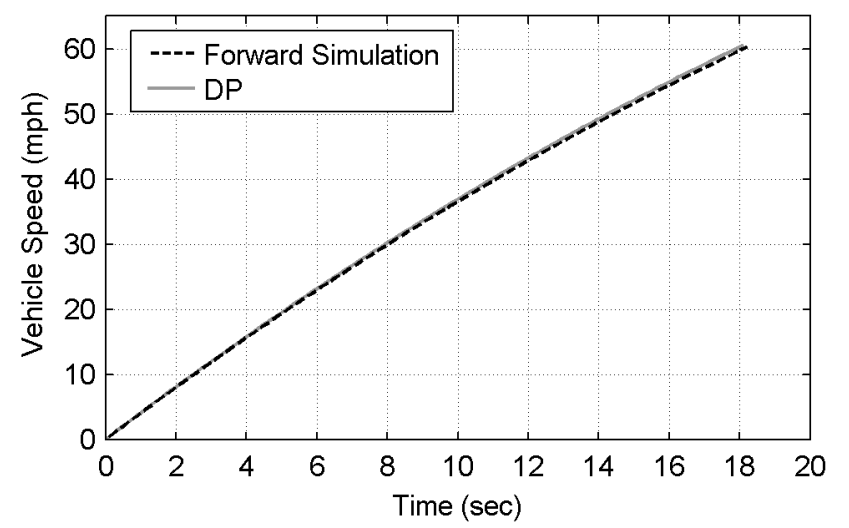

FIGURE 7. SPEED TRAJECTORIES TO ACCELERATE FROM 0 TO 60 MPH IN THE SPLIT MODE.
Forward simulations are also applied on Mode 1 and 3 to find the shortest time to accelerate from 0 to $60 \mathrm{mph}$ (Mode 2 is always less powerful than Mode 4, and thus not shown); the results are shown in Figure 8. The results turn out to be somewhat counter-intuitive; the two 1-DOF modes (Mode 1 and 3) accelerate much faster than Mode 4, and furthermore, Mode 3 performs slightly better than Mode 1 because Mode 3 has both the engine and the battery powering MG2 whereas Mode 1 has only the battery powering MG2.

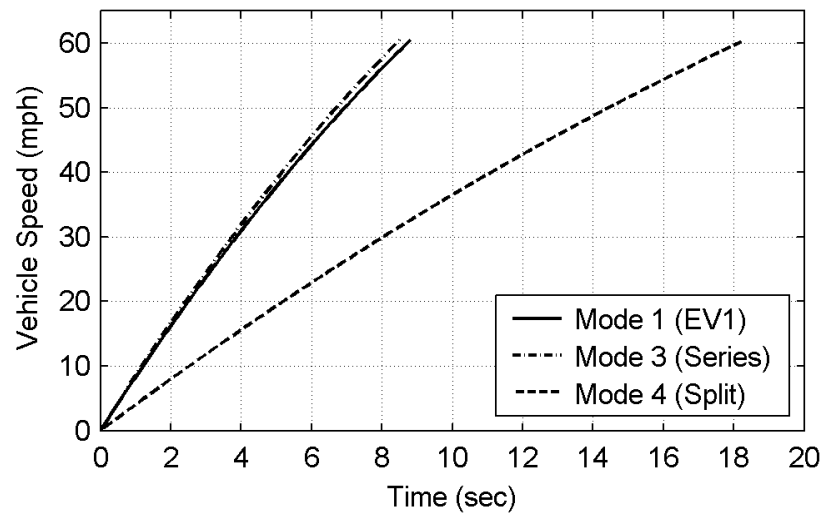

FIGURE 8. SPEED TRAJECTORIES UNDER WIDE-OPEN THROTTLE ACCELERATION FROM 0 TO 60 MPH USING DIFFERENT OPERATION MODES

The reason that 1-DOF modes can outperform a (2-DOF) split mode is because 1-DOF modes use clutches to ground a node on the PG, and uses this grounded node to provide torque. In this paper, we assume the grounding clutch capacity is unlimited. When the node is not grounded, its torque is limited by the powertrain component. This PG-grounding feature is actually used by General Motors on 2-PG hybrid SUVs to provide better towing capability [24].

In summary, the drivability performance of HEV configurations is evaluated by simulating the wide-openthrottle acceleration scenario from 0 to $60 \mathrm{mph}$. Analysis of rotational accelerations on the lever diagram provides insights about the best acceleration strategy in the spit mode, which can be used in forward simulations, so we do not need to conduct DP optimizations to evaluate drivability. Furthermore, it is observed that the drivability can be improved significantly by grounding one node of the PG.

\section{Step 4: Fuel Economy Optimization}

The optimization problem to minimize the fuel consumption over a pre-defined driving cycle is formulated and solved by DP to establish the benchmark performance of each configuration candidate. The parameters, control/state variables and objective function shown in Table 2, Table 3, and Eq. (10) are used in DP to optimize the fuel economy of the Chevy Volt. 
TABLE 2. PARAMETERS OF POWERTRAIN COMPONENTS FOR THE CHEVY VOLT [25]

\begin{tabular}{cccc}
\hline Parameters & MG1 & MG2 & Engine \\
\hline Max. Speed (rpm) & 6,000 & 9,500 & 4,000 \\
Max. Torque (Nm) & 200 & 370 & $102 @ 4,000 \mathrm{rpm}$ \\
Max. Power (kW) & 55 & 110 & 43 \\
\hline Planetary Gear Ratio (R:S) & \multicolumn{3}{c}{$2.24: 1$} \\
Final Drive Ratio & \multicolumn{3}{c}{2.16} \\
\hline
\end{tabular}

TABLE 3. STATES \& CONTROL VARIABLES FOR FUEL ECONOMY OPTIMIZATION

\begin{tabular}{cc}
\hline States & Range \\
\hline$\omega_{M G 1}$ & $-6000: 500: 6000(\mathrm{rpm})$ \\
\hline$S O C$ & $0.4: 0.01: 0.7(-)$ \\
\hline Control Variables & Range \\
\hline$T_{e}$ & $0: 10: 100(\mathrm{Nm})$ \\
\hline$T_{M G 1}$ & $-200: 10: 200(\mathrm{Nm})$ \\
\hline Mode & $1,2,3,4($ discrete $)$ \\
\hline \multirow{2}{*}{$\min \left\{J=\sum_{k=0}^{N-1}\left(F C_{k}+\beta \cdot \Delta\right.\right.$ Mode $\left.\left._{k}+\gamma \cdot \Delta S O C_{k}\right)\right\}$}
\end{tabular}

where $F C$ is the rate of fuel consumption, $\triangle M o d e$ is the mode shifting event, and $\triangle S O C$ is the change in battery SOC. The mode shifting penalty ensures that the number of mode shifts is not excessive, and the SOC penalty ensures that the vehicle uses the battery efficiently in pure-electric modes (Modes 1 and 2 ). The penalty weights, $\beta$ and $\gamma$, are chosen to be small so that
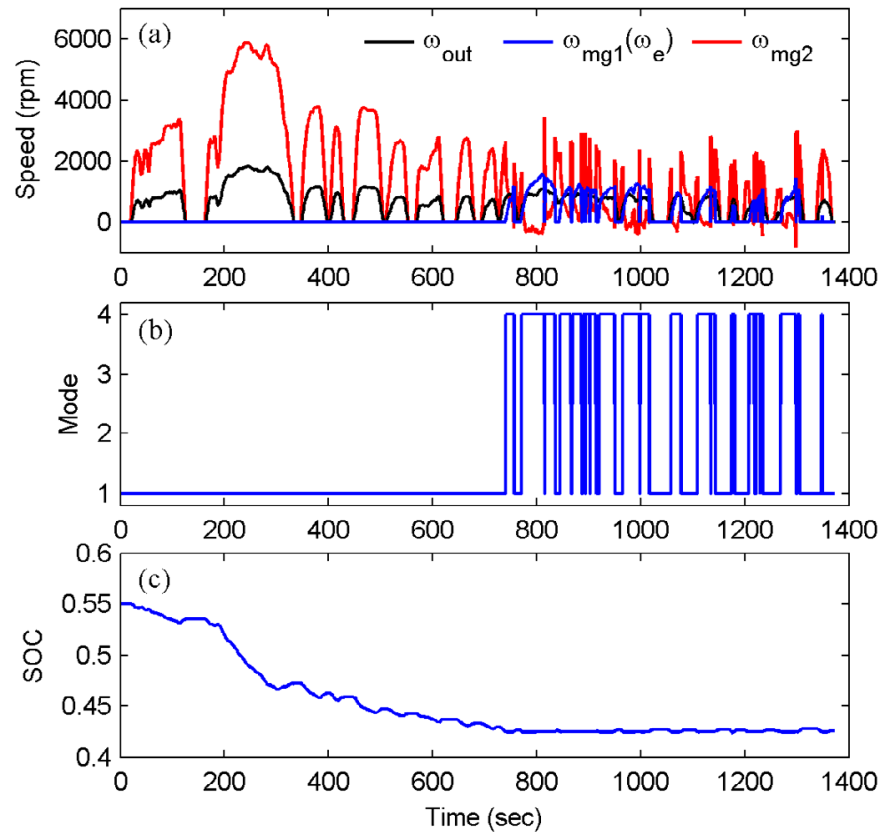

FIGURE 9. THE CHEVY VOLT IN FUDS CYCLE the fuel consumption is still the dominating term in the optimization. Figure 9 shows the optimal solution for the FUDS driving cycle, and the optimal fuel consumption is 125.8 g.

\section{CASE-STUDY ILLUSTRATING THE DESIGN METHODOLOGY}

This section shows results of applying the proposed design methodology on two particular configuration candidates: the output-split configuration used by the Chevy Volt and the input-split configuration used by the Toyota Prius.

\section{The Output-Split Configuration Used by Chevy Volt}

This configuration candidate is shown in Figure 10-(a), which is derived from Figure 3, and its drivability $(0-60 \mathrm{mph}$ in 8.5 seconds using Mode 3) and optimal fuel consumption (125.8 $\mathrm{g}$ in the FUDS cycle) were shown in the previous section. The following presents the results when the design iteration is performed.

The mode shift in Figure 9-(b) shows that this 4-mode output-split configuration uses mainly Modes 1 and 4 in the FUDS cycle, which inspires a simplified design with only one clutch to allow the configuration to switch only between Mode 1 and Mode 4. This simplified configuration is named Volt and shown in Figure 10-(b).

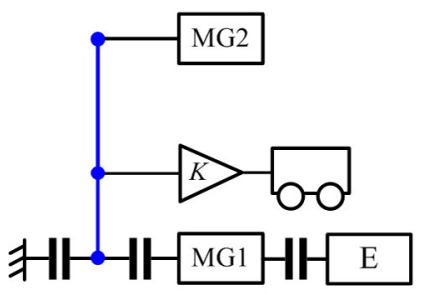

(a) The original Volt

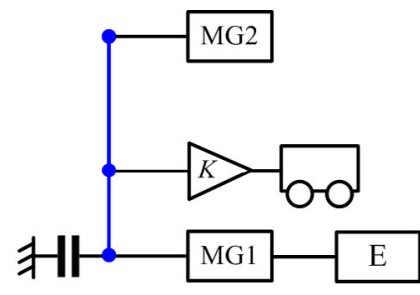

(b) The Volt
FIGURE 10. THE ORIGINAL VOLT AND THE SIMPLIFIED DESIGN "VOLT"”.

Table 4 summarizes the performances of the original Volt and the simplified Volt. Notice that the simplified Volt has the drivability slightly deteriorated because it uses Mode 1 for acceleration which is not as powerful as Mode 3 (as shown in Figure 8). The Volt also performs slightly worse than the original Volt in the highway HWFET cycle because it has fewer operation modes.

TABLE 4. PERFORMANCE OF VOLT \& VOLT'

\begin{tabular}{ccccc}
\hline \multirow{2}{*}{ Vehicle } & Drivability & & \multicolumn{2}{c}{ Fuel Consumption } \\
\cline { 2 - 3 } \cline { 5 - 5 } & $\mathbf{0 - 6 0} \mathbf{~ m p h}$ & & FUDS & HWFET \\
\hline Volt (four modes) & $8.5 \mathrm{~s}$ & & $125.8 \mathrm{~g}$ & $320.5 \mathrm{~g}$ \\
\hline Volt $^{-}$(Modes 1 \& 4) & $8.8 \mathrm{~s}$ & $125.8 \mathrm{~g}$ & $323.2 \mathrm{~g}$ \\
\hline
\end{tabular}




\section{The Input-Split Configuration Used by Toyota Prius}

This configuration candidate is shown in Figure 11 with all seven clutches. Notice that the real Toyota Prius, shown in Figure 13-(a), has only one split mode and does not use any clutch.

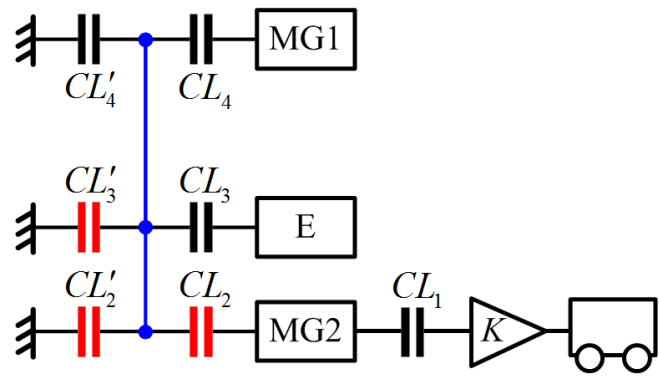

FIGURE 11. THE INPUT-SPLIT CONFIGURATION USED BY THE TOYOTA PRIUS WITH ADDITIONAL CLUTCHES.

The analysis of Step 1 is shown in Table 5. Clutch $C L_{1}$ is dismissed automatically because the output shaft is assumed to be always connected to the PG and the standing-still battery charging never happened. It is, again, found that only four out of the eight operation modes are useful, and only the three red clutches $\left(C L_{2}, C L_{2}{ }^{\prime}\right.$, and $\left.C L_{3}{ }^{\prime}\right)$ are needed to achieve those four modes.

TABLE 5. CLUTCH STATES \& OPERATION MODES OF THE INPUT-SPLIT CONFIGURATION USED BY TOYOTA PRIUS.

\begin{tabular}{lccc}
\hline \multicolumn{1}{c}{ Mode } & $\boldsymbol{C L}_{\mathbf{2}}$ & $\boldsymbol{C L}_{\mathbf{3}}$ & $\boldsymbol{C L}_{\mathbf{4}}$ \\
\hline $1\left(\mathrm{EV}_{1}\right)$ & 0 & 0 & 1 \\
\hline $2\left(\mathrm{EV}_{2}\right)$ & 0 & 1 & 1 \\
\hline 3 (Series) & 1 & 0 & 1 \\
\hline 4 (Split) & 1 & 1 & 1 \\
\hline $5\left(=\mathrm{EV}_{1}\right)$ & 0 & 0 & 0 \\
\hline $6\left(=\mathrm{EV}_{1}\right)$ & 0 & 1 & 0 \\
\hline 7 (Infeasible) & 1 & 0 & 0 \\
\hline 8 (No EVT) & 1 & 1 & 0 \\
\hline
\end{tabular}

Description: " 0 " means the clutch is open; " 1 " means the clutch is closed.

Step 1 concludes that this input-split configuration can have up to four operation modes, and then drivability is evaluated and fuel economy is optimized for this initial 4-mode input-split configuration using the parameters listed in Table 6 . The results are shown in Table 7 and Figure 12. It is found that the 4-mode Prius outperforms the original Prius in both drivability and fuel consumption, because its clutch provides the PG-grounding feature to boost the acceleration performance, and the multiple operation modes provide more flexibility to improve fuel economy.
TABLE 6. PARAMETERS OF POWERTRAIN COMPONENTS FOR THE TOYOTA PRIUS

\begin{tabular}{cccc}
\hline Parameters & MG1 & MG2 & Engine \\
\hline Max. Speed (rpm) & 12,000 & 12,000 & 4000 \\
Max. Torque (Nm) & 200 & 200 & $102 @ 4000 \mathrm{rpm}$ \\
Max. Power (kW) & 50 & 50 & 43 \\
\hline Planetary Gear Ratio (R:S) & \multicolumn{3}{c}{$2.6: 1$} \\
Final Drive Ratio & 3.95 \\
\hline
\end{tabular}

TABLE 7. PERFORMANCE OF THE INPUT-SPLIT CONFIGURATION USED BY PRIUS

\begin{tabular}{ccccc}
\hline Vehicle & Drivability & & \multicolumn{2}{c}{ Fuel Consumption } \\
\cline { 2 - 2 } & $\mathbf{0 - 6 0} \mathbf{~ m p h}$ & & FUDS & HWFET \\
\hline 4-Mode Prius & $7.8 \mathrm{~s}$ & $101.8 \mathrm{~g}$ & $310.8 \mathrm{~g}$ \\
\hline Prius (only Mode 4) & $10.8 \mathrm{~s}$ & $120.0 \mathrm{~g}$ & $320.6 \mathrm{~g}$ \\
\hline Prius $^{+}$(Modes 2 and 4) & $7.8 \mathrm{~s}$ & $103.8 \mathrm{~g}$ & $312.6 \mathrm{~g}$ \\
\hline
\end{tabular}
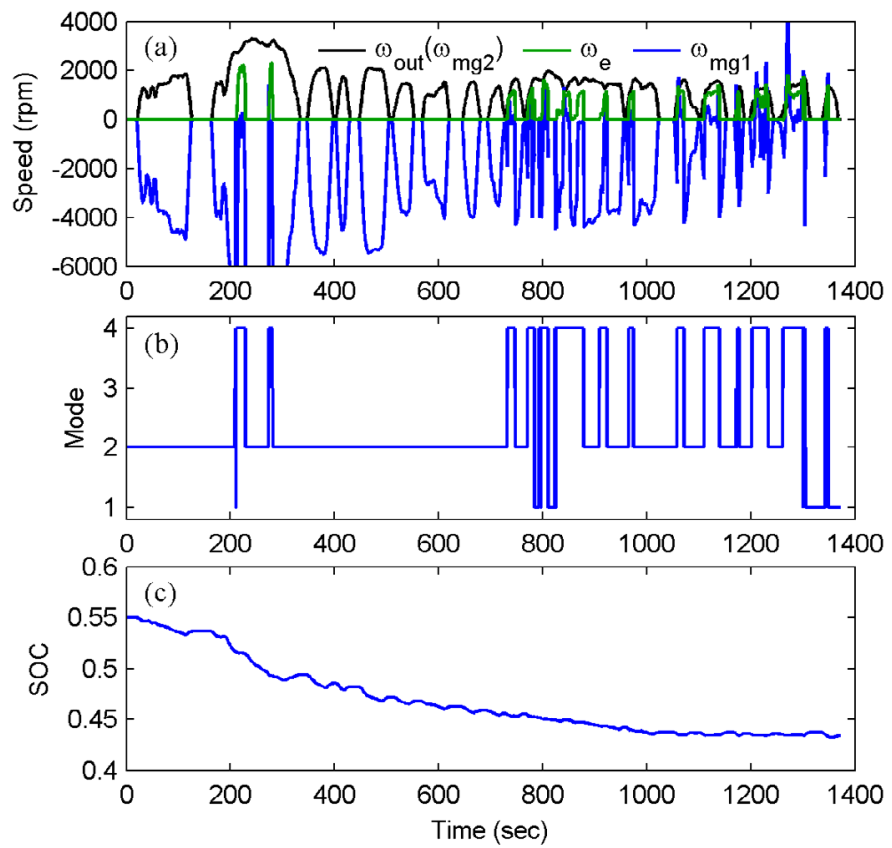

FIGURE 12. THE 4-MODE PRIUS IN THE FUDS CYCLE.

The design iteration is then performed. The shift sequence in Figure 12-(b) shows that Modes 2 and 4 are used more frequently than the other modes, which inspires the alternative 1-clutch-2-mode design, the Prius ${ }^{+}$, shown in Figure 13-(b). The performances of the Prius + are also listed in Table 7. It can be seen that the Prius ${ }^{+}$performs better than the original Prius and is almost as good as the 4-mode Prius. The Prius ${ }^{+}$inherits the strong drivability from the 4-mode Prius because it still possesses the PG-grounding feature. Its fuel economy is close to the 4-mode Prius because its two operation modes provide 
enough flexibility to operate efficiently in both the FUDS and HWFET cycles.

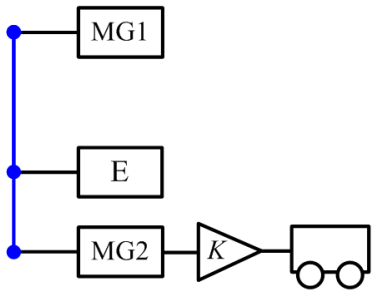

(a) The original Prius

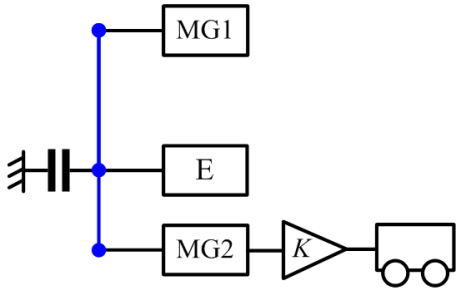

(b) Prius +

\section{FIGURE 13. THE ORIGINAL PRIUS AND THE IMPROVED "PRIUS+".}

\section{CONCLUSION}

We present a comprehensive design process to systematically examine all of the 12 single-PG configuration candidates of power-split hybrid powertrain. The design process has four steps: Step 1 analyzes the clutch locations on the PG to identify useful operation modes; Step 2 generates dynamic models in the state space representations; Step 3 evaluates the drivability, i.e. the acceleration performance via forward simulations; and Step 4 uses DP to optimize the fuel economy. The execution of the design process on two configuration candidates, the output-split configuration used in the Chevy Volt and the input-split configuration used in the Toyota Prius, are reported in this paper.

Step 1 shows that all the 12 configuration candidates can have four useful operation modes by adding three clutches on the PG. Starting with the 3-clutch-4-mode layout, dynamic models for the configuration candidates can be generated (Step 2). In Step 3, strategies derived from analyzing rotational accelerations on the lever diagram are used in forward simulations to evaluate the $0-60 \mathrm{mph}$ acceleration. The simulations show that grounding one node on the PG can improve the acceleration performance. Step 4 uses the dynamic programming (DP) technique to optimize the fuel consumption so that the benchmark performance can be accessed. The DP solutions show that, in general, having more operation modes improves fuel economy because of the added flexibility. However, not all modes are used frequently in all configuration candidates. The mode that does not improve the drivability or rarely used in the DP solution can be eliminated, which can reduce the powertrain complexity and cost. The design methodology helps us to identify alternative designs, the Volt and Prius ${ }^{+}$, which are derived from the original Volt and Prius. Both of these two new designs have one clutch, which enables two operation modes.

The Volt is reduced from the Volt, in which only one of the three clutches is kept. This clutch allows the Volt to operate in Mode $1\left(E_{1}\right)$ and Mode 4 (split). The simplified Volt has the same fuel economy in the FUDS cycle as the original Volt, and it has slightly reduced performance in drivability and fuel economy in the HWFET cycle. The Prius+ is an improved version of the original Prius, in which one clutch is added. In addition to the split mode, this additional clutch allows the Prius $^{+}$to operate in a pure-electric mode (Mode 2). The extra flexibility improves the drivability (due to the PG-grounding feature) and fuel economy. The results on these two configuration candidates show that there is a tradeoff between design complexity and performance, a1 34770889487871

nd the design trade-off can be studied systematically using the proposed design process.

\section{REFERENCES}

[1] Alternative Fuels and Advanced Vehicles Data Center, Data, Analysis, and Trends: Vehicle-HEV Sales by Model, [online] 2010 http://www.afdc.energy.gov/afdc/data/ vehicles.html (Accessed: 1/9/2012).

[2] Hybridcars website, December 2010 Dashboard: Year End Tally, [online] $2011 \mathrm{http} / / / w w w . h y b r i d c a r s . c o m / h y b r i d-$ clean-diesel-sales-dashboard/december-2010.html (Accessed: 1/8/2012).

[3] J. Liu and H. Peng, "A systematic design approach for two planetary gear split hybrid vehicles," Vehicle System Dynamics, vol. 48, pp. 1395-1412, 2010.

[4] X. Zhang, C.-T. Li, D. Kum and H. Peng, "Prius+ and Volt-: Configuration Analysis of Power-Split Hybrid Vehicles with a Single Planetary Gear," IEEE Transactions on Vehicular Technology, under review.

[5] C.-T. Li and H. Peng, "Optimal configuration design for hydraulic split hybrid vehicles," in American Control Conference, Baltimore, MD, 2010, pp. 5812-5817.

[6] C. Mansour and D. Clodic, "Dynamic modeling of the electro-mechanical configuration of the Toyota Hybrid System series/parallel power train," International Journal of Automotive Technology, vol. 13, pp. 143-166, 2012.

[7] J. Liu and H. Peng, "Modeling and control of a power-split hybrid vehicle," IEEE Transactions on Control Systems Technology, vol. 16, pp. 1242-1251, 2008.

[8] F. Freudenstein and A. Yang, "Kinematics and statics of a coupled epicyclic spur-gear train," Mechanism and Machine Theory, vol. 7, pp. 263-275, 1972.

[9] H. Benford and M. Leising, "The lever analogy: a new tool in transmission analysis," SAE Paper, 810102, 1981.

$[10]$ K. Cheong, P. Li and T. Chase, "Optimal design of powersplit transmission for hydraulic hybrid passenger vehicles," in American Control Conference, San Francisco, CA, 2011, pp. 3295-3300.

[11] B. Conlon, "Comparative analysis of single and combined hybrid electrically variable transmission operating modes," SAE Paper, 2005-01-1162, 2005.

[12]D. Hermance, "Toyota Hybrid System," in SAE TOPTEC Conference, Albany, NY, 1999.

[13]N. Jalil, N. Kheir and M. Salman, "A rule-based energy management strategy for a series hybrid vehicle," in 
American Control Conference, Albuquerque, New Mexico, 1997, pp. 689-693.

[14] G. Pagalelli, S. Delprat, T. Guerra, J. Rimaux and J. Santin, "Equivalent consumption minimization strategy for parallel hybrid powertrains," in 55th IEEE Vehicular Technology Conference, 2002, pp. 2076-2081.

[15]A. Sciarretta, M. Back and L. Guzzella, "Optimal control of parallel hybrid electric vehicles," IEEE Transactions on Vehicular Technology, vol. 12, pp. 352-363, 2004.

[16] S. Stockar, V. Marano, G. Rizzoni and L. Guzzella, "Optimal control for plug-in hybrid electric vehicle applications," in American Control Conference, Baltimore, MD, 2010, pp. 5024-5030.

[17] S. Delprat, J. Lauber, T. M. Guerra and J. Rimaux, "Control of a parallel hybrid powertrain: optimal control," IEEE Transactions on Vehicular Technology, vol. 53, pp. 872-881, 2004.

[18] S. Delprat, T. M. Guerra and J. Rimaux, "Control strategies for hybrid vehicles: optimal control," in 56th IEEE Vehicular Technology Conference, Vancouver, Canada, 2002, pp. 1681-1685.

[19]N. Kim, S. Cha and H. Peng, "Optimal control of hybrid electric vehicles based on Pontryagin's minimum principle," IEEE Transactions on Control Systems Technology, vol. 19, pp. 1279 - 1287, 2010.

[20]C.-C. Lin, H. Peng, J. Grizzle and J.-M. Kang, "Power management strategy for a parallel hybrid electric truck," IEEE Transactions on Control Systems Technology, vol. 11, pp. 839-849, 2003.

[21] J. Liu and H. Peng, "Control optimization for a power-split hybrid vehicle," in American Control Conference, Minneapolis, Minnesota, 2006, pp. 466-471.

[22]C. Mansour and D. Clodic, "Optimized energy management control for the Toyota Hybrid System using dynamic programming on a predicted route with short computation time," International Journal of Automotive Technology, vol. 13, pp. 309-324, 2012.

[23] A. Sciarretta and L. Guzzella. (2005) Control of hybrid electric vehicles. IEEE Control Systems Magazine. pp. 6070.

[24] T. Grewe, B. Conlon and A. Holmes, "Defining the general motors 2-mode hybrid transmission," SAE Paper, 2007-010273, 2007.

[25] K. Rahman, M. Anwar, S. Schulz, E. Kaiser, P. Turnbull, S. Gleason, B. Given and M. Grimmer, "The Voltec 4ET50 Electric Drive System," SAE Paper, 2011-01-03, 2011. 